\title{
A DIPLEXER BASED ON THE SPATIAL FILTERING PROPERTY OF PLANAR ANISOTROPIC TRANSMISSION-LINE METAMATERIALS
}

\author{
Joshua K. H. Wong ${ }^{(1)}$, Keith G. Balmain ${ }^{(2)}$, and George V. Eleftheriades ${ }^{(3)}$ \\ The Edward S. Rogers Sr. Department of Electrical and Computer Engineering \\ University of Toronto \\ 10 King's College Road, Toronto, Ontario, M5S 3G4, Canada \\ ${ }^{(1)}$ Email: joshua@waves.utoronto.ca \\ ${ }^{(2)}$ Email: balmain@waves.utoronto.ca

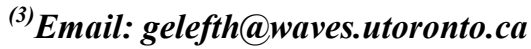

\section{INTRODUCTION}

The objective of this work is to investigate the possible use of metamaterials to achieve frequency diplexing. The metamaterial of interest is a planar anisotropic metamaterial proposed by Balmain et al. in [1]. It was shown in [2] that this metamaterial is magnetically anisotropic and could be described by a diagonal permeability tensor. For a certain range of frequencies, two of the three diagonal elements of the permeability tensor become opposite in sign. Under this condition, plane-wave propagation is governed by a hyperbolic dispersion equation and the metamaterial could be referred to as a hyperbolic metamaterial [3]. As a result, under point-source excitation, resonance cones form as a consequence of the singularity associated with the characteristic surface of the hyperbolic dispersion equation. Since the resonance cone angle, which describes the direction of power flow [4], varies with the frequency of the propagating wave, the phenomenon may properly be termed "spatial filtering." The diplexer presented in this work exploits this inherent spatial filtering property of the hyperbolic metamaterial to achieve frequency diplexing.

\section{THEORY OF OPERATION}

A schematic of the diplexer is shown in Fig. 1. The diplexer consists of two functional blocks: a spatial filter and a reciprocal lens. Regarding its operation, one sees that a wide-band signal is fed through the input port into a spatial filter. The spatial filter separates the input signal into its component spectra where each component propagates in a different direction. The spatially separated signals are then fed into the second block called the reciprocal lens. The reciprocal lens is thus named because unlike a conventional lens, it does not focus the incident resonance cones but rather, refracts them into a single direction for all angles of incidence. Thus the reciprocal lens enables control over the directions of the edge-reflected cones. If the incident cones impinge on the output ports perpendicularly, the reflected cones are directed back to the input port without incurring multiple variously-directed reflections and resultant crosstalk within the grid.

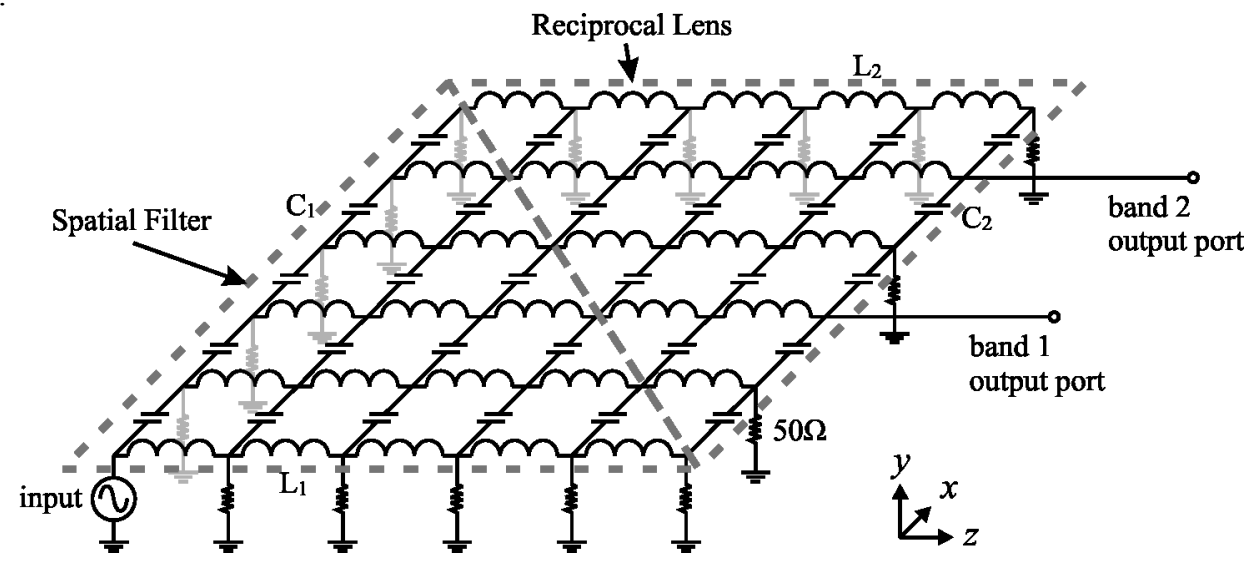

Fig. 1. Schematic of a diplexer realized on a two-region hyperbolic metamaterial with corner feed and resistive terminations. The per-unit inductances and capacitances for the spatial filter and the reciprocal lens are $\mathrm{L}_{1}=4.6 \mathrm{nH}, \mathrm{C}_{1}=2.2 \mathrm{pF}, \mathrm{L}_{2}=1.0 \mathrm{nH}$, and $\mathrm{C}_{2}=13.7 \mathrm{fF}$ with the self-inductance of the interconnecting transmission lines taken into consideration. 


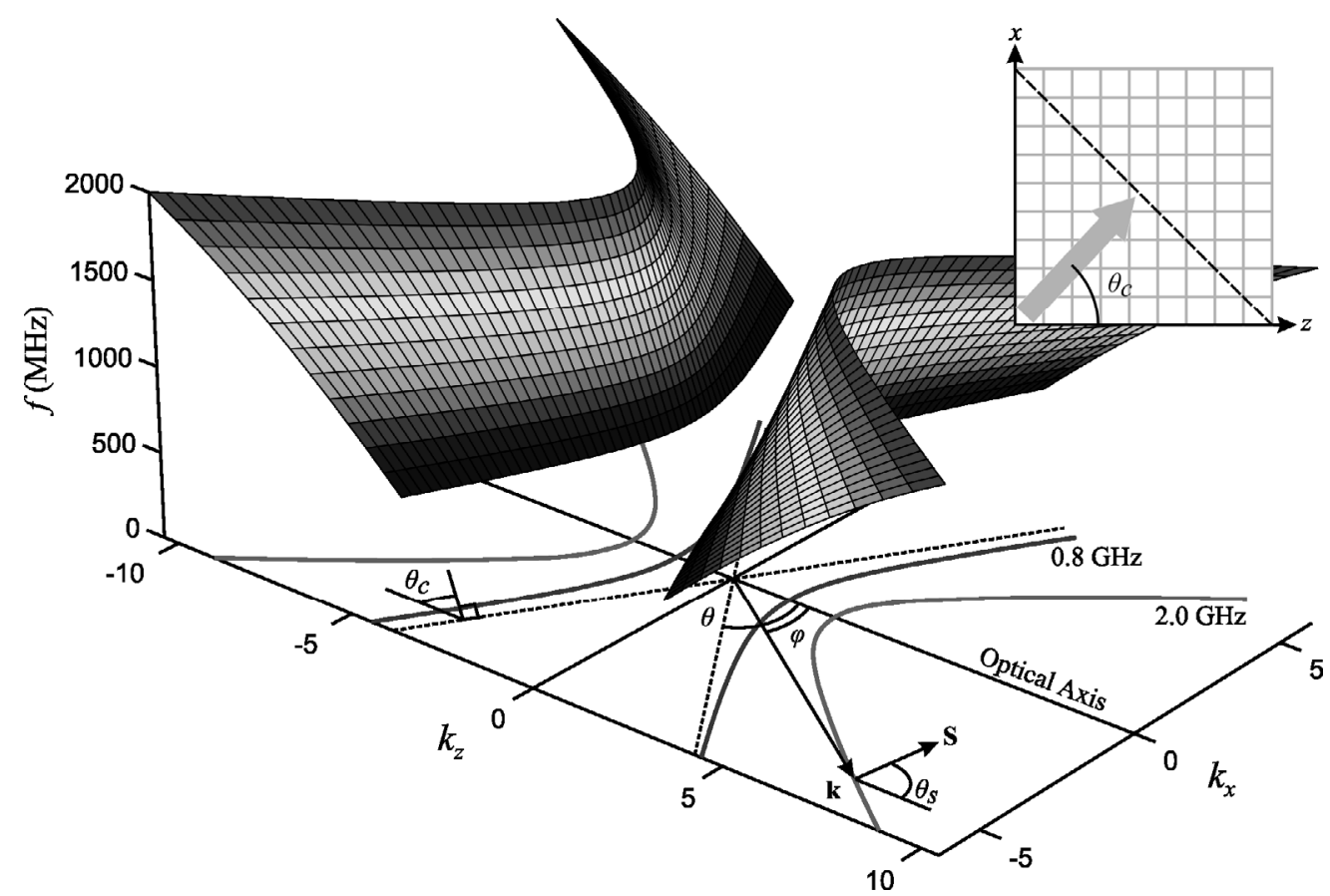

(a) Spatial Filter

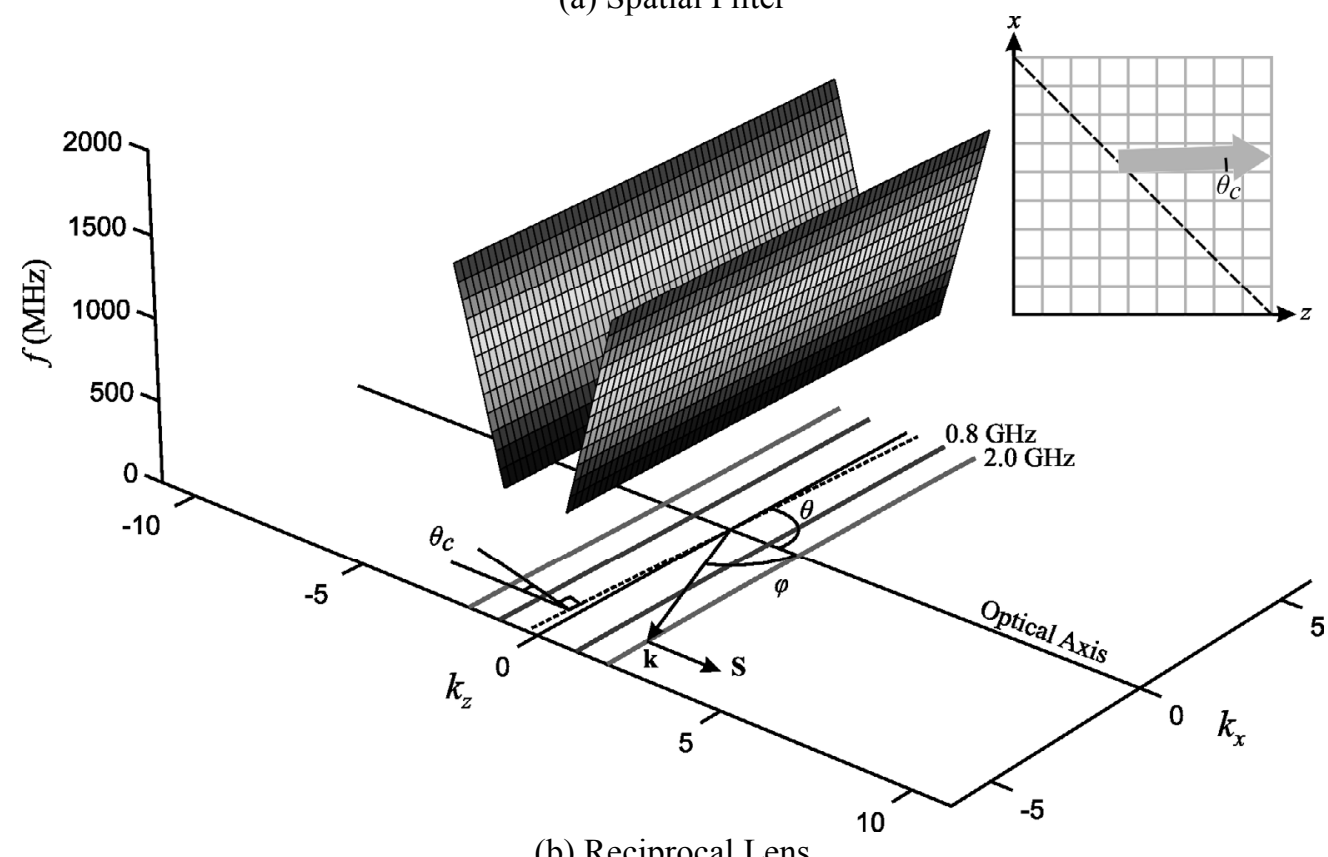

(b) Reciprocal Lens

Fig. 2. Wavenumber surfaces from $800 \mathrm{MHz}$ to $2000 \mathrm{MHz}$ with contour projections for $800 \mathrm{MHz}$ and $2000 \mathrm{GHz}$. The insets show the resonance cone orientation with respect to the anisotropic grid.

The resulting wavenumber surfaces of the spatial filter and the reciprocal lens are shown in Fig. 2. Contour projections of the dispersion surface at $800 \mathrm{MHz}$ and $2.0 \mathrm{GHz}$ are projected at the bottom of the graph which show the hyperbolic characteristics of the dispersion relation. Furthermore, plane-wave propagation is confined spatially to the regions $\varphi<\theta$. For $\varphi>\theta$, the wave number is imaginary and propagation is evanescent. The Poynting vector $\mathbf{S}$ indicates the direction of power flow and is confined to the interior of the conical regions where $\left|\tan \theta_{s}\right|<\tan \theta_{c}$. The limiting angle $\theta_{c}$, or the resonance cone angle, for the Poynting vector is thus defined by the perpendicular of the asymptotes (the dashed lines in Fig. 2) with respect to the optical axis as shown in Fig. 2. The insets show how the resonance cone is oriented with respect to the anisotropic grid. 


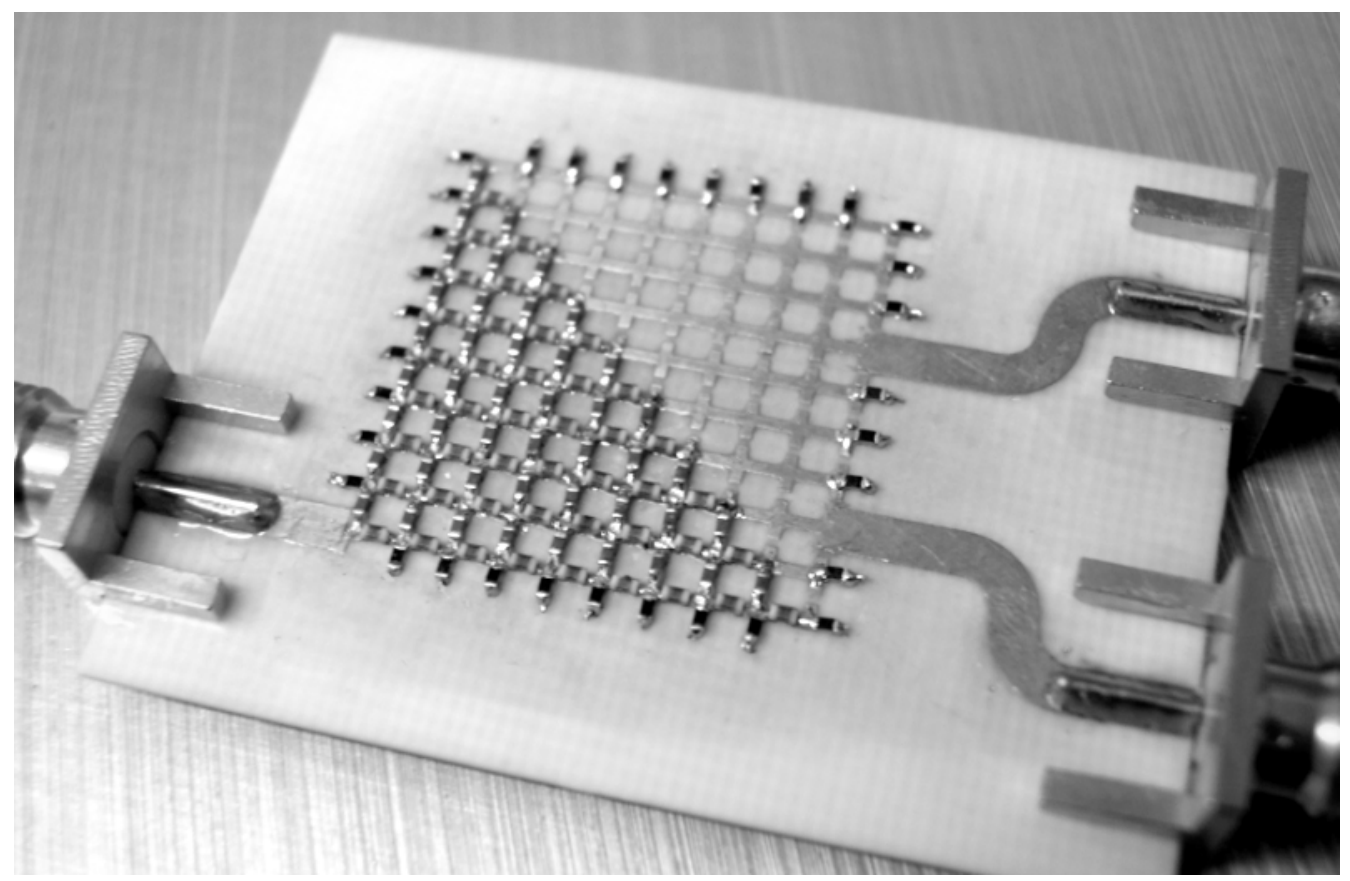

Fig. 3. Photograph of the completed prototype circuit with feed lines and SMA connectors.

The spatial filter was designed in such a way that the opening angle of the asymptotes $\theta$ varies with the operating frequency whereas for the reciprocal lens $\theta$ was designed such that it remains relatively constant over the entire range of the operating frequency. For the spatial filter, the cone angle increases monotonically with increasing frequency, from $\theta_{c}=27.6^{\circ}$ at $f=800 \mathrm{MHz}$ to $\theta_{c}=57.8^{\circ}$ at $f=2000 \mathrm{MHz}$. In other words, the low frequency components of a wide-band signal would propagate with a smaller cone angle than the high frequency components. For the reciprocal lens, the cone angle variation is much smaller. In this case, the cone angle varies from $\theta_{c}=1.1^{\circ}$ at $f=800 \mathrm{MHz}$ to $\theta_{c}=2.7^{\circ}$ at $f=2000 \mathrm{MHz}$.

\section{RESULTS}

The completed prototype circuit is shown in Fig. 3. Chip-type surface mount inductors and capacitors were used in the spatial filter. For the reciprocal lens, microstrip gap capacitors were used. The termination resistors are located along the edges of the grid on the top side of the board, and vias are used to connect the resistors to the ground plane. The vias are implemented with plated copper with a plating thickness of around $16 \mu \mathrm{m}$. To accommodate the size of the input and output port connectors, $50 \Omega$ microstrip transmission lines were added to each port. The grid itself is $20 \mathrm{~mm}$ by $20 \mathrm{~mm}$ and consists of 9 by 9 cells.

The diplexer is designed to operate from $800 \mathrm{MHz}$ to $2000 \mathrm{MHz}$ with the lower passband from $869 \mathrm{MHz}$ to $894 \mathrm{MHz}$ and the upper passband from $1930 \mathrm{MHz}$ to $1990 \mathrm{MHz}$. Fig. 4 shows the resonance cone simulations of the diplexer in the two passbands. The simulations employed a full-wave, thin-wire moment-method program [5] to study the power flow within the diplexer. To study the power flow in the experiments, we used a vector network analyzer to measure the vertical electric field just above each conductor intersection. The linear magnitude of the analyzer's $\mathrm{S}_{21}$ response is measured which is approximately proportional to the grid-to-ground voltage. The resulting surface scan measurements for the two passbands are shown in Fig. 5a and Fig. 5b. As shown in Fig. 4 and Fig. 5, the experiments and the simulations are in excellent agreement with each other. In the lower passband, the resonance cone refracts positively whereas in the upper passband, it refracts negatively. The resonance cones impinge upon the edge of the grid perpendicularly in both passbands as designed. Furthermore, we can see there is no visible power leakage in the form of beam spreading between the two output ports.

\section{CONCLUSION}

The diplexer realised on a planar anisotropic transmission-line metamaterial has been constructed and tested. The near-field measurements show the expected spatial filtering property and resonance cone phenomena, including positive 


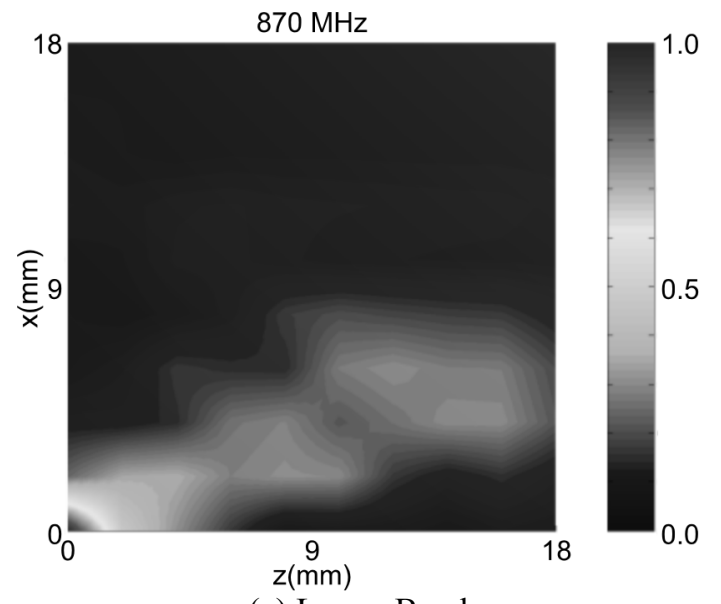

(a) Lower Band

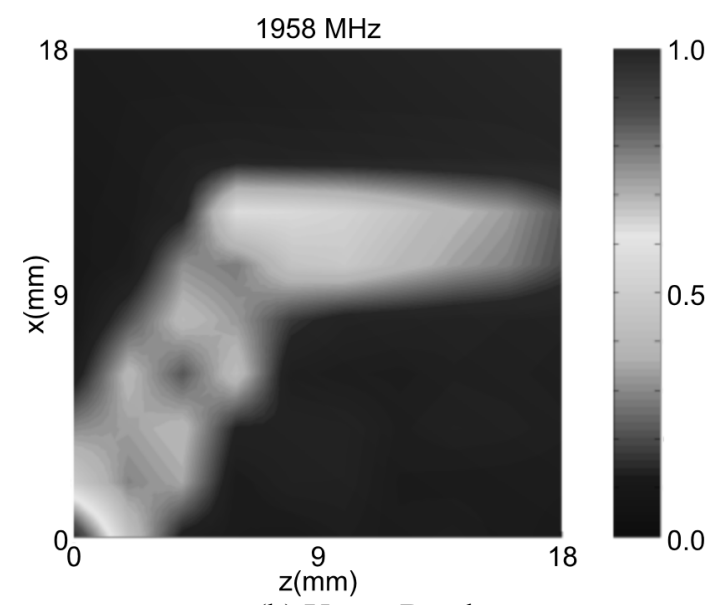

(b) Upper Band

Fig. 4. Simulations showing the normalized grid-to-ground voltage magnitude.

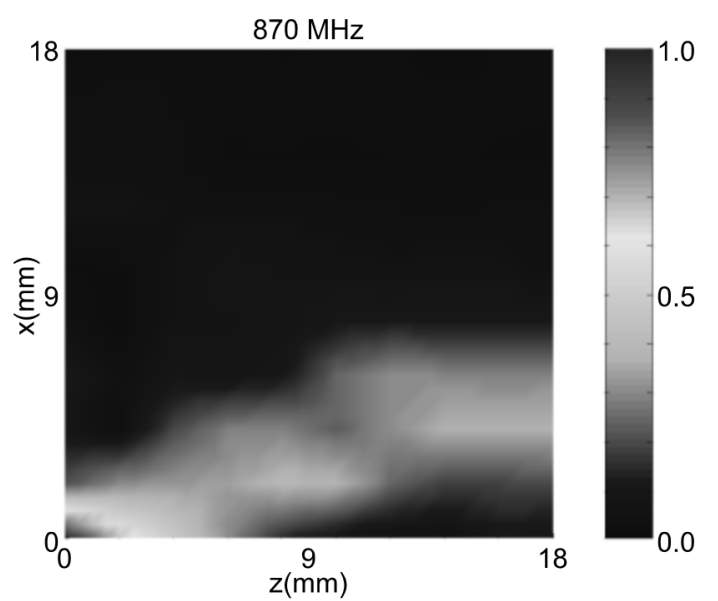

(a) Lower Band

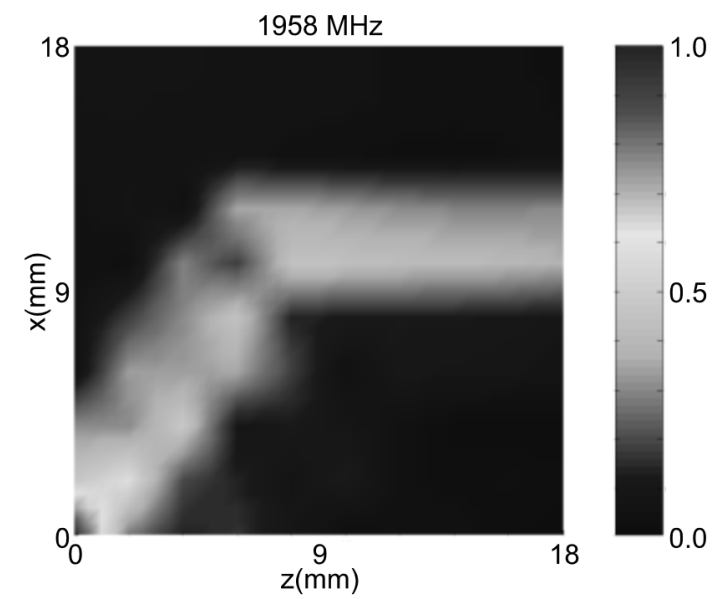

(b) Upper Band

Fig. 5. Surface scan experiments showing the normalized $S_{21}$ magnitude measured across the surface of the grid.

and negative refraction at an interface. Furthermore, the use of a diagonal interface enables a well-defined transition from one medium to another which would have otherwise introduced distortion in the unit cells around the transition region [4].

Although in the present context where we consider the planar anisotropic transmission-line metamaterial to be used as a diplexer, the design concept may very well be extended into that of a multiplexer by adding more output ports. The spatial filtering properties of the metamaterial add a new dimension to filter design philosophy that is not conceivable in conventional "one dimensional" circuit filter.

\section{REFERENCES}

[1] K. G. Balmain, A. A. E. Lüttgen, and P. C. Kremer, "Resonance cone formation, reflection, refraction, and focusing in a planar anisotropic metamaterial," IEEE Antennas and Wireless Propagation Letters, vol. 1, no. 7, pp. 146-149, 2002.

[2] J. K. H. Wong, "Spatial filtering in anisotropic transmission-line metamaterials," Master's thesis, Dept. of Electrical and Computer Engineering, University of Toronto, Toronto, 2004.

[3] O. F. Siddiqui and G. V. Eleftheriades, "Negative refraction and focusing in hyperbolic transmission-line periodic grids," IEEE Trans. Microwave Theory and Techniques, vol. 53, no. 1, pp. 396-403, Jan. 2005.

[4] K. G. Balmain, A. A. E. Lüttgen, and P. C. Kremer, "Power flow for resonance cone phenomena in planar anisotropic metamaterials," IEEE Trans. Antennas and Propagation, vol. 51, no. 10, pp. 2612-2618, Oct. 2003.

[5] M. A. Tilston, and K. G. Balmain, "A multiradius, reciprocal implementation of the thin-wire moment method," IEEE Trans. Antennas and Propagation, vol. 38, no. 10, pp. 1636-1644, Oct. 1990. 